\title{
Tunable focalizers: phase conjugate pairs
}

Jorge Ojeda-Castañeda, Cristina Gómez-Sarabia

Jorge Ojeda-Castañeda, Cristina M. Gómez-Sarabia, "Tunable focalizers: phase conjugate pairs," Proc. SPIE 11207, Fourth International Conference on Applications of Optics and Photonics, 112072L (3 October 2019); doi: 10.1117/12.2530686

SDIE Event: IV International Conference on Applications of Optics and Photonics (AOP 2019), 2019, Lisbon, Portugal 


\title{
Tunable Focalizers: Phase Conjugate Pairs
}

\author{
Jorge Ojeda-Castañeda ${ }^{*}$, a and Cristina M. Gómez-Sarabia ${ }^{b}$ \\ ${ }^{a}$ Electronics Dept., Engineering Division, University of Guanajuato, 36885 Salamanca, Mexico \\ ${ }^{\mathrm{b}}$ Digital Arts, Engineering Division, University of Guanajuato, 36885 Salamanca, Mexico
}

\begin{abstract}
We discuss the use of pairs of optical masks for setting tunable optical focalizers and for implementing controllable absorption masks. For phase-only masks, one element of a given pair has a complex amplitude transmittance that is equal to the complex conjugate of the other element. For the absorption masks, we use a suitable attenuation offset. Then, from the attenuation offset value, one element has the opposite absorption profile that the other element. These methods are useful for generating varifocal lenses, governable prisms, tunable axicons, controllable axilenses, for tuning field depth, and for controlling Super Gaussian beams.
\end{abstract}

Keywords: Tunable Prisms and Lenses, Governable Field Depth, Controllable Diffraction Efficiency, Optical Deflectors, Tunable Spherical Aberration, Beam Shapers, and Switchable Attenuation Coefficients.

\section{INTRODUCTION}

There are several competing technologies for implementing varifocal lenses; as well as for producing phase-only masks with governable diffraction efficiencies. Some of the previous technologies can conveniently be applied to the generation of tunable absorption masks. In Table 1, we show a roadmap of our current contribution.

\begin{tabular}{|c|c|c|c|}
\hline Tunable capabilities: & Optical Path Differences & Diffraction Efficiencies & Attenuation Coefficient \\
\hline Phase - only & Prisms, Axicons, Lenses & Gratings and Zone Plates & -- \\
\hline Amplitude - only & --- & Zero Order Devices & $\begin{array}{c}\text { Gaussians Beams } \\
\text { and Super Gaussians }\end{array}$ \\
\hline
\end{tabular}

Table 1. Roadmap of the use of conjugate optical pairs.

In Table 1, along the first line, we describe the capability of a given pair of elements for controlling: the optical path differences, or the diffraction efficiencies, or the attenuation coefficient, respectively. Along line 2, we indicated that phase-only pairs are useful for implementing tunable prisms, axicons and lenses; as well as for controlling the diffraction efficiencies of 1-D sinusoidal phase gratings and of 2-D, radially symmetric, sinusoidal zone plates. And along line 3, we indicate that amplitude pairs are useful for controlling the damping factor of absorption masks, with a specific attenuation profile.

In section 2, we discuss the use of conjugated phase pairs for controlling, either by an in-plane displacement or by an inplane rotation, the optical path difference. In section 3, we illustrate the previous result for setting a large family of focalizers; and for tuning field depth without modifying the size of the pupil aperture. Then, in section 4, we show that the same method helps for tuning the diffraction efficiency of deflectors, in the form of 1-D sinusoidal phase gratings and of sinusoidal phase zone plates. Next, in section 5, we discuss the use of an offset attenuation value for proposing masks that have complementary absorption variations, with respect to an offset value. Both types of pairs may be useful for implementing nonconventional apodizers. Finally, in section 6, we summarize our contribution.

*jojedacas@ugto.mx; phone 52464 555-1234

Fourth International Conference on Applications of Optics and Photonics, edited by

Manuel F. M. Costa, Proc. of SPIE Vol. 11207,112072L · C) 2019 SPIE

CCC code: $0277-786 \mathrm{X} / 19 / \$ 21 \cdot$ doi: $10.1117 / 12.2530686$ 


\section{TUNING OPTICAL PATH DIFFERENCE: PHASE CONJUGATED PAIRS}

In Figure 1, we depict the use of two transparent optical elements, each of them with a cubic phase profile, for implementing a lens with tunable optical power, as proposed simultaneously by Lohmann [1-3] and by Alvarez [4].

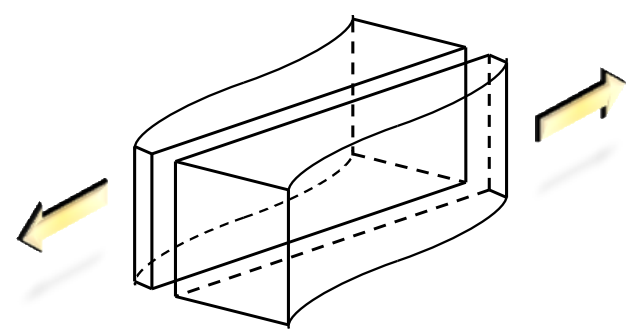

Figure 1. Optical arrangement of two phase conjugated masks, with cubic phase profile, for implementing a lens with tunable optical power.

The working principle for setting varifocal lenses, by employing a pair of transparent optical masks, is here denoted as the use of phase conjugate pairs. This working principle can be expressed in terms of the calculus of differences, as follows [5]. For the first optical element, the 1-D complex amplitude transmittance is

$$
\mathrm{T}_{1}(\mathrm{x})=\exp [\mathrm{i} 2 \pi \mathrm{p} \psi(\mathrm{x})] .
$$

In Eq.(1) the lower case letter p denotes the optical path difference. And the Greek letter $\psi$ represents the 1-D phase profile function. For the second optical element, of the pair, the complex amplitude transmittance is

$$
\mathrm{T}_{2}(\mathrm{x})=\mathrm{T}_{1} *(\mathrm{x})=\exp [-\mathrm{i} 2 \pi \mathrm{p} \psi(\mathrm{x})]
$$

Eq.(2) defines the phase conjugate mask. Now, if one uses the two above elements in close contact, and after one introduces an in-plane lateral displacement, between the two elements (say by a distance y), the overall amplitude transmittance is

$$
T(x ; y)=T_{1}(x+y / 2) T_{1} *(x-y / 2)=\exp \{\text { i } 2 \pi p[\psi(x+y / 2)-\psi(x-y / 2)]\} .
$$

In a first order approximation, Eq.(3) can be written as

$$
\mathrm{T}(\mathrm{x} ; \mathrm{y})=\exp [\mathrm{i} 2 \pi(2 \mathrm{p} y) \Delta \psi(\mathrm{x})]
$$

In Eq.(4) the first difference of the function $\psi(\mathrm{x})$ is denote as $\Delta \psi(\mathrm{x})$. For some examples, as in the case for the Lohmann - Alvarez lenses, the first order approximation is an exact expression. See reference [5] for other examples. It is apparent from Eq.(4) that one can control the optical path difference, of the overall complex amplitude transmittance, only by introducing an in-plane lateral displacement.

Inspired by Lohmann contribution to varifocal optics [6], as well as by Dyson grating descriptions [7] and Bryngdahl contribution to nonconventional interferograms [8], some of us proposed the use of a pair of helical phase masks for 
generating a large family set of refractive focalizers [9-11]. For diffractive focalizers see the work of Bernet et al. [12] and that Grewe et al. $[13,14]$.

We have noted that with radial polar coordinate $r$, and for practically any radial function $R(r)$, it is convenient to generate (as a first phase element of a pair) the complex amplitude transmittance

$$
\mathrm{T}_{1}(\mathrm{r}, \varphi)=\exp [\mathrm{i} 2 \pi \mathrm{p} R(\mathrm{r})(\varphi / 2 \pi)]
$$

In Eq.(5) we denote as $\varphi$ the polar angle, which is trivially contained inside the interval $(0,2 \pi)$. The second element, of the phase conjugated pair, has the following complex amplitude transmittance

$$
\mathrm{T}_{2}(\mathrm{r}, \varphi)=\mathrm{T}_{1} *(\mathrm{r}, \varphi)=\exp [-\mathrm{i} 2 \pi \mathrm{p} R(\mathrm{r})(\varphi / 2 \pi)]
$$

Eq.(6) defines the phase conjugate mask.

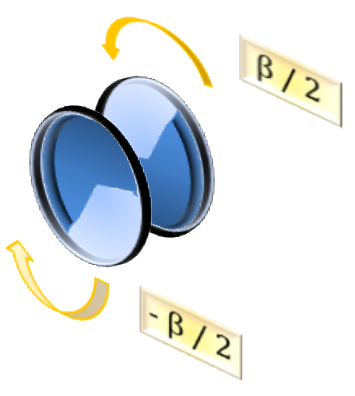

Figure 2. Optical arrangement of two helical phase conjugated masks, for implementing a lens with tunable optical power.

Now, as depicted in Figure 2, if one uses the two above elements in close contact, and after one introduces an in-plane rotation between the two elements (say by an angle $\beta$ ), the overall amplitude transmittance is

$$
\mathrm{T}(\mathrm{r}, \beta)=\mathrm{T}_{1}(\mathrm{r}, \varphi+\beta / 2) \mathrm{T}_{1} *(\mathrm{r}, \varphi+\beta / 2)=\exp [\text { i } 2 \pi(\mathrm{p} \beta / 2 \pi) \mathrm{R}(\mathrm{r})]
$$

It is clear from Eq.(7) that by introducing an in-plane rotation, between the elements of the pair, one can tune the optical path difference of almost any radially symmetric phase profile. In what follows we discuss some examples, including the generation of wave front aberrations.

\section{TUNABLE OPTICAL FOCALIZERS}

The axicon is the classical circularly symmetric, refractive element that is able to concentrate the irradiance distribution along the optical axis [15]. In Figure 3, we display the interferogram of a lens and that of an axicon, as well as the interferograms associated to the helical versions of a lens and of an axicon.

There are other optical elements that have a similar behavior to that of an axicon, one of them is known as the axilens [16-18], and other variation incorporates purposely spherical aberration [19]. For this latter case, the overall complex amplitude transmittance in Eq.(7) becomes 


$$
\mathrm{T}(\mathrm{r} ; \beta)=\exp \left[\text { i } 2 \pi(\mathrm{p} \beta / 2 \pi)(\mathrm{r} / \mathrm{L})^{4}\right] \cdot \operatorname{circ}(\mathrm{r} / \mathrm{L}) \text {. }
$$
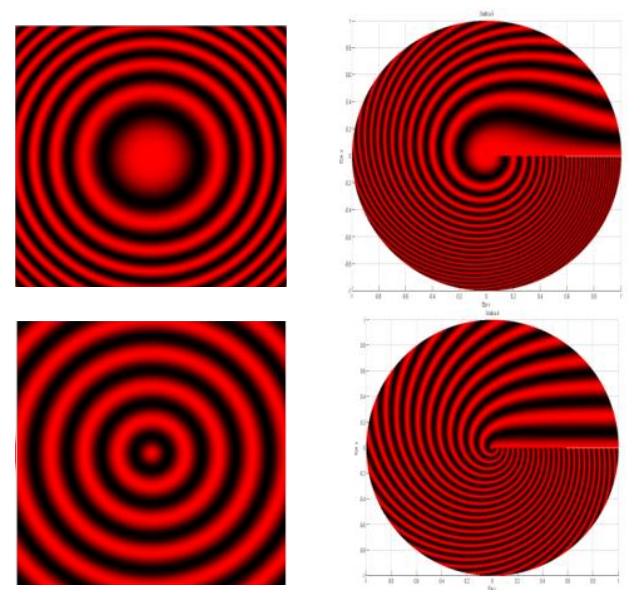

Figure 3. Interferograms of 2-D phase distributions that have linear variation in $r$. However, in (a) the phase variation is independent of the polar angle, and in (b) the phase distribution also varies linearly with the polar angle.

In Eq.(8) the upper case letter $\mathrm{L}$ denotes the radius of the pupil aperture encircling the lens. But now the optical path difference is proportional to the aberration coefficient for primary spherical aberration, $\mathrm{W}_{\mathbf{4 , 0}}$, that is

$$
\mathrm{p}=\left(\mathrm{W}_{\mathbf{4 , 0}} / \lambda\right)
$$

In Eq.(9) the Greek letter lambda stands for the wavelength of the optical radiation. By substituting Eq.(9) in Eq.(8) we have that the overall complex amplitude transmittance is

$$
\mathrm{T}\left(\mathrm{r} ; \beta ; \mathrm{W}_{\mathbf{4}, \mathbf{0}}\right)=\exp \left[\mathrm{i}\left(\beta \mathrm{W}_{\mathbf{4 , 0}} / \lambda\right)(\mathrm{r} / \mathrm{L})^{\mathbf{4}}\right] .
$$

It is apparent from Eq.(10) that by introducing an in-plane rotation, by an angle $\beta$, one can control the amount of spherical aberration in an optical system.

It is straightforward to show, by employing McCutchen's theorem [20], that the axial irradiance distribution is similar to the irradiance distribution of the Fresnel diffraction pattern of a caused slit aperture [21].

Before ending this section, it is relevant to note the following. For extending field depth, one employs phase masks that under the autocorrelation operation generate optical transfer functions, with low sensitivity to focus errors. Hence, at each spatial frequency the autocorrelation operation (using a single phase mask) is equivalent to a lateral displacement of a pair of phase conjugated masks. Then, under the convolution operation, a single cubic mask (one element of the Lohmann - Alvarez lens) behaves as tunable lens for the optical transfer function of the optical system [22].

\section{OPTICAL DEFLECTORS: TUNNING THE DIFFRACTION EFFICIENCIES}

Here, we show that the concept of phase conjugated pairs is useful for controlling the diffraction efficiencies of optical deflectors, as depicted in Figures 4 and 5. To that end, next we analyze the use of a pair of phase conjugate, sinusoidal gratings. The first grating has the following complex amplitude transmittance 


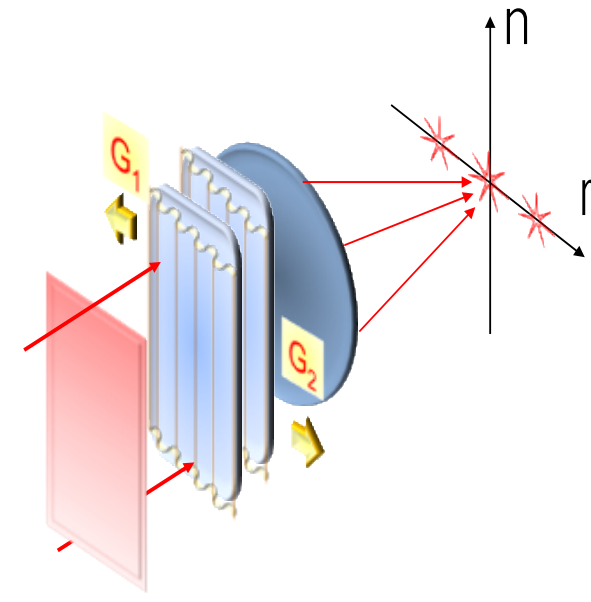

Figure 4. Optical arrangement of two sinusoidal gratings, for controlling diffraction efficiency.

In Eq.(11) the period of the sinusoidal phase grating is d. The complex amplitude transmittance of the second grating is

$$
\mathrm{T}_{2}(\mathrm{x})=\exp [-\mathrm{i} 2 \pi \mathrm{p} \cos (2 \pi \mathrm{x} / \mathrm{d})]
$$

As before, if one places these phase gratings in close contact, and after one introduces an in-plane lateral displacement, between the two gratings (by a distance y), the overall amplitude transmittance is

$$
\mathrm{T}(\mathrm{x} ; \mathrm{y})=\mathrm{T}_{1}(\mathrm{x}+\mathrm{y} / 2) \mathrm{T}_{1} *(\mathrm{x}-\mathrm{y} / 2)=\exp \{\mathrm{i} 2 \pi[2 \mathrm{p} \sin (\pi \mathrm{y} / \mathrm{d})] \sin (2 \pi \mathrm{x} / \mathrm{d}\} .
$$

By using the Anger-Jacobi relationship, one can rewrite Eq.(13) as

$$
\mathrm{T}(\mathrm{x} ; \mathrm{y})=\sum_{\mathrm{k}=-\infty}^{\infty} \operatorname{Jm}[4 \pi \mathrm{p} \sin (\pi \mathrm{y} / \mathrm{d})] \exp [\mathrm{i} 2 \pi \mathrm{m}(\mathrm{x} / \mathrm{d})]
$$

In Eq.(14) we denote as Jm (.) the Bessel functions of the first kind and of integer order m. From Eq.(14), one can obtain that the diffraction efficiency, at the m-th diffraction focus, here denoted as the Greek letter "eta". That is,

$$
\eta_{\mathrm{m}}=\left|\mathrm{J}_{\mathrm{m}}[4 \pi \mathrm{p} \sin (\pi \mathrm{y} / \mathrm{d})]\right|^{2}
$$

From Eq.(15) we claim that one can control the diffraction efficiency of the m-th diffraction order, simply by introducing a lateral displacement, $y$, between the conjugate pair of sinusoidal phase gratings. 
As depicted in Figure 5, the above results ca be extended to control the diffraction efficiency of sinusoidal phase zone plates. For this purpose, the conjugate phase pair is the following. The first complex amplitude transmittance is now a helical version of a sinusoidal phase, zone plate. That is,

$$
\mathrm{T}_{1}(\mathrm{r}, \varphi)=\exp \left[\mathrm{i} 2 \pi \mathrm{p}(\varphi / 2 \pi) \sin \left(2 \pi(\mathrm{r} / \mathrm{d})^{2}\right]\right.
$$

Eq.(16) defines the complex conjugate of the first complex amplitude transmittance . For the second mask, the complex complex amplitude transmittance is

$$
\mathrm{T}_{2}(\mathrm{r}, \varphi)=\exp \left[-\mathrm{i} 2 \pi \mathrm{p}(\varphi / 2 \pi) \sin \left(2 \pi(\mathrm{r} / \mathrm{d})^{2}\right]\right.
$$

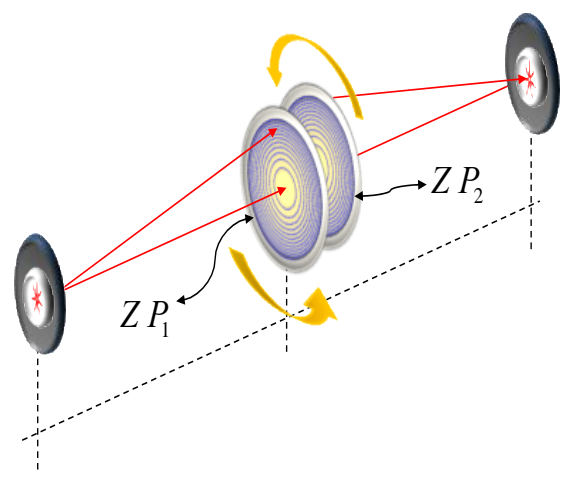

Figure 5. Optical arrangement of two sinusoidal zone plates, for controlling diffraction efficiency of the $\mathrm{m}$-th focus.

As before, if one uses the two above elements in close contact, and after one introduces an in-plane rotation between the two elements (say by an angle $\beta$ ), the overall amplitude transmittance is

$$
\mathrm{T}(\mathrm{r} ; \beta)=\mathrm{T}_{1}(\mathrm{r}, \varphi+\beta / 2) \mathrm{T}_{1} *(\mathrm{r}, \varphi+\beta / 2)=\exp \left[\mathrm{i} 2 \pi(\mathrm{p} \beta / 2 \pi) \sin \left(2 \pi(\mathrm{r} / \mathrm{d})^{2}\right]\right.
$$

Again, by using the Anger-Jacobi relationship, one can rewrite Eq.(18) as

$$
\mathrm{T}(\mathrm{r} ; \beta)=\sum_{\mathrm{k}=-\infty}^{\infty} \operatorname{Jm}(2 \mathrm{p} \beta) \exp \left[\mathrm{i} 2 \pi \mathrm{p}(\mathrm{r} / \mathrm{d})^{2}\right]
$$

In Eq.(19) we denote as Jm (.) the Bessel functions of the first kind and of integer order m. From Eq.(19), one can obtain the diffraction efficiency, at the m-th diffraction focus, is denoted as the Greek letter "eta". That is,

$$
\eta_{\mathrm{m}}=\left|\mathrm{J}_{\mathrm{m}}(2 \mathrm{p} \beta)\right|^{2}
$$


Therefore, from Eq.(19), we conclude that one can control the diffraction efficiency of the m-th diffraction focus, simply by introducing an in-plane rotation, by an angle $\beta$, between the complex conjugate pair of sinusoidal phase zone plates.

\section{GOVERNABLE ABSORPTION: COMPLEMENTARY AMPLITUDE PAIRS}

For amplitude-only masks, there is not a straightforward manner to set a complex conjugate operation. However, in what follows, we show that it a possible to stablish an offset attenuation value. Then, one can have both positive absorption departures, and negative absorption departures, from the offset attenuation value. As an example of this procedure, we depicted in Figure 6, the design of two complementary masks (curve in blue and curve in red), which act as a pair for controlling the half-width of a Gaussian transmittance [23]. Other attenuation masks can also be designed using the same optical procedure [24].

(a)

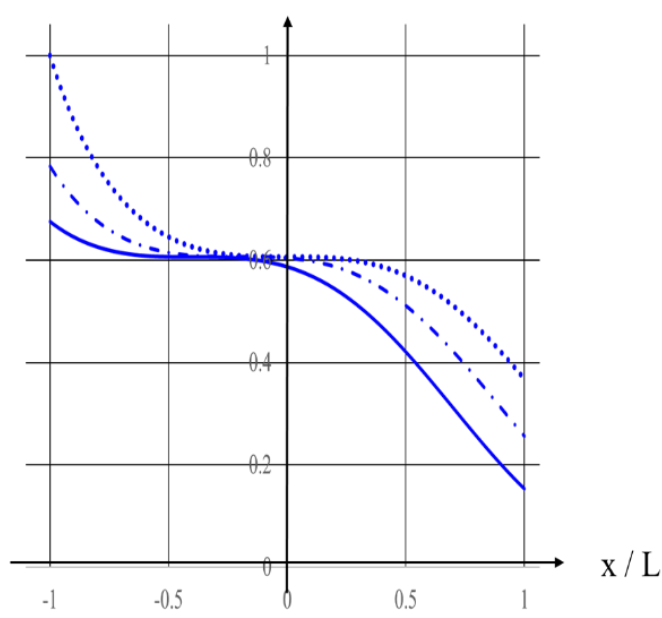

(b)

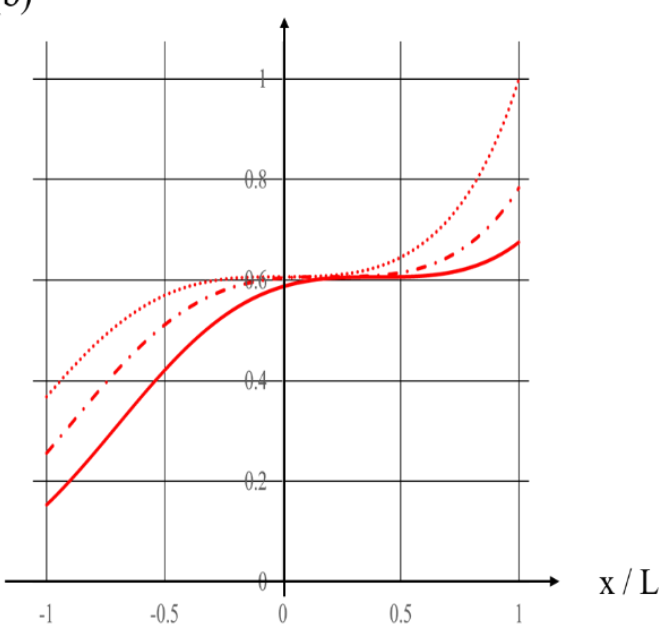

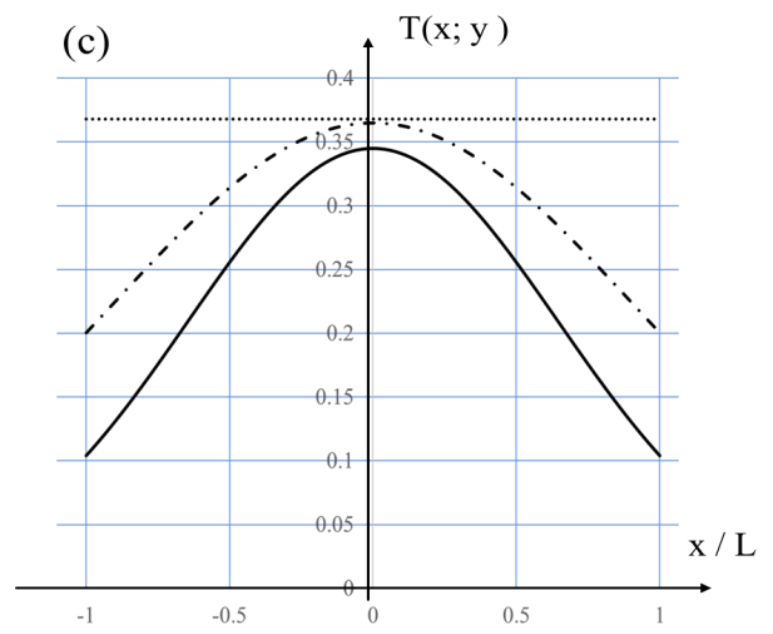

Figure 6. Amplitude transmittance, inside the pupil aperture, of two masks that are complementary. In (a) the blue curves describe the amplitude transmittance variations of the first mask. In (b) the same, but in red, for the second mask. In (c) the same, but in black, for the overall amplitude transmittance. The dotted curves are for zero lateral displacement, $y=0$. The broken curves are for a lateral displacement, $y=L / 5$. And the full curves are for a lateral displacement, $\mathrm{y}=(2 / 5) \mathrm{L}$. 
For the first element of the pair, the amplitude transmittance is the curve in blue, in Figure 6. In mathematical terms

$$
\mathrm{T}_{1}(\mathrm{x})=\exp \left\{-\mathrm{a}\left[1+(\mathrm{x} / \mathrm{L})^{3}\right] \operatorname{rect}(\mathrm{x} / 2 \mathrm{~L})\right.
$$

In Eq.(21) we denote with the lower case letter a, the absorption coefficient of the amplitude masks. The value of the absorption coefficient specifies the offset attenuation value at the origin $\mathrm{x}=0$. The mask extends from $\mathrm{x}=-\mathrm{L}$ to $\mathrm{x}=\mathrm{L}$. This interval represents the pupil aperture, which is here represented by the rectangular function in Eq.(21). The maximum transmittance value, that is equals to unity, is located at $\mathrm{x}=-\mathrm{L}$. The minimum transmittance value is located at $\mathrm{x}=\mathrm{L}$.

For the second element of the pair, the amplitude transmittance is

$$
\mathrm{T}_{2}(\mathrm{x})=\exp \left\{-\mathrm{a}\left[1-(\mathrm{x} / \mathrm{L})^{3}\right] \operatorname{rect}(\mathrm{x} / 2 \mathrm{~L}) .\right.
$$

From Eq.(22) we note that the maximum transmittance value, that is equals to unity, is located at $\mathrm{x}=\mathrm{L}$. And the minimum transmittance value is located at $\mathrm{x}=-\mathrm{L}$. In Figure 7 we show the overall transmittance, if we employ the two amplitude masks as a pair and we introduce a lateral displacement, say by a distance y, between the masks. Under this conditions, the overall complex amplitude transmittance is

$$
\mathrm{T}(\mathrm{x} ; \mathrm{y})=\mathrm{T}_{1}(\mathrm{x}+\mathrm{y} / 2) \mathrm{T}_{2}(\mathrm{x}-\mathrm{y} / 2)=\exp (-2 \mathrm{a}) \exp \left[-(2 \mathrm{a} \mathrm{y} / \mathrm{L})(\mathrm{x} / \mathrm{L})^{2}\right] \operatorname{rect}(\mathrm{x} / 2 \mathrm{~L})
$$

From Eq.(23) we claim that by a lateral displacing, between the above two amplitude masks, one can control the halfwidth of a Gaussian attenuation profile. Next, we consider the equivalent in absorption masks of the helical phase variations in section 3. But now, the two complementary masks have amplitude helical variations. In mathematical terms, the amplitude transmittance of the first masks is

$$
\mathrm{T}_{1}(\mathrm{r}, \varphi)=\exp \{-\mathrm{a}[1+\mathrm{R}(\mathrm{r})(\varphi / 2 \pi)]\} \operatorname{circ}(\mathrm{r} / \mathrm{L})
$$

In Eq.(24) we employ polar coordinates, $r$ and $\varphi$, and we denote the radial variations with the function $R(r)$. The finite size of the mask is expressed by using the circ function, which is equal to unity if $\mathrm{L} \geq \mathrm{r}$. Otherwise, the circ function is equal to zero. The amplitude transmittance of the second masks is

$$
\mathrm{T}_{2}(\mathrm{r}, \varphi)=\exp \{-\mathrm{a}[1-\mathrm{R}(\mathrm{r})(\varphi / 2 \pi)]\} \operatorname{circ}(\mathrm{r} / \mathrm{L})
$$

Since the masks are passive filters then, from Eq.(24), we have that 5Now, as depicted in Figure 7, if one uses the two above masks in close contact, and after one introduces an in-plane rotation between the two masks (say by an angle $\beta$ ), the overall amplitude transmittance is

$$
\mathrm{T}(\mathrm{r} ; \beta)=\mathrm{T}_{1}(\mathrm{r}, \varphi+\beta / 2) \mathrm{T}_{1} *(\mathrm{r}, \varphi+\beta / 2)=\exp \{-\mathrm{a}[1+\mathrm{R}(\mathrm{r})(\beta / 2 \pi)]\} \operatorname{circ}(\mathrm{r} / \mathrm{L})
$$

In Figure 7, we depict our proposal for the generation of Super Gaussian absorption masks, with controllable attenuation coefficient. Hence, in Eq.(27) we set $\mathrm{m}=5$. 


$$
\mathrm{T}(\mathrm{r} ; \beta)=\exp \left\{-\mathrm{a}\left[1+(\mathrm{r} / \mathrm{L})^{\mathrm{m}}(\beta / 2 \pi)\right]\right\} \operatorname{circ}(\mathrm{r} / \mathrm{L}) .
$$

(a)

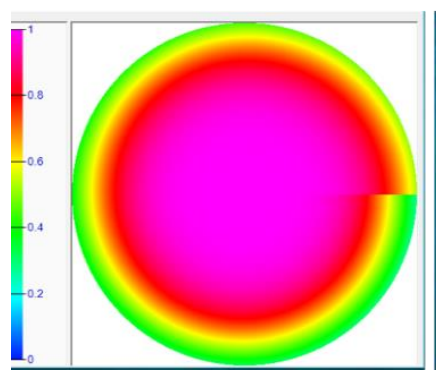

(b)

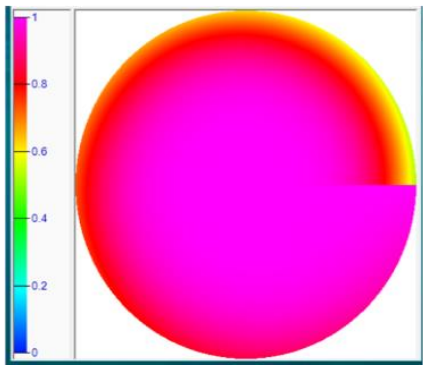

(c)

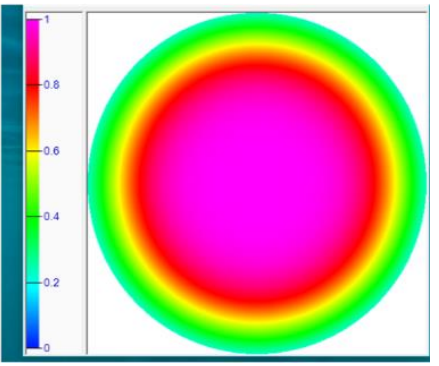

Figure 7 Amplitude transmittance, inside the pupil aperture, of two complementary helical absorption masks. At the left-hand, we plot the 3-D, absorption graph of the first mask. At the center, the same as before, but for the second mask. And at the right-hand, we plot the generated Super Gaussian absorbing mask.

From Eq.(26) we claim that, by an in-plane rotation, by an angle $\beta$, one can control the absorption coefficient of attenuation masks that have hyper Gaussian attenuation profiles. Here it is relevant to note that the phase conjugate pairs and the amplitude complementary pairs can be used simultaneously in a tandem arrangement [26].

\section{FINAL REMARKS}

We have discussed a generalization of the Lohmann-Alvarez lens, which is here recognized aa a phase conjugate pair. We have shown that by using other phase conjugate pairs, one can control the optical path difference, only by introducing an in-plane lateral displacement.

Furthermore, we have indicated that, under the convolution operation, a single element of the phase conjugated pair behaves as tunable lens for the optical transfer function of the optical system.

We have noted that the phase conjugate pair concept is useful for controlling the diffraction efficiency of the m-th diffraction order; to this end one only needs to introduce a lateral displacement, between a conjugate pair of sinusoidal phase gratings.

Then, we have discussed a 2-D variation of the phase conjugate pair, for governing the optical path difference with radial symmetry. To this goal, we have employed polar coordinates; and we have shown that indeed, by an in-plane rotation, one can control optical path differences of practically any radial function $\mathrm{R}(\mathrm{r})$.

This 2-D variation of the phase conjugate pair is useful for tuning the diffraction efficiency of the $\mathrm{m}$-th diffraction focus, of sinusoidal phase zone plates; which form a suitable phase conjugate pair. Moreover, we have indicated that by introducing an in-plane rotation, one can control the amount of spherical aberration in an optical system.

Then, we have shown that one can have both positive absorption departures, and negative absorption departures, from the offset attenuation value. This key observation allows one for controlling the half-width of a Gaussian attenuation profile.

We have shown that a 2-D variation of the amplitude complementary masks, each having helicoidal amplitude variations, is useful for setting a large family of radially symmetric, beam shapers, with tunable damping coefficient. 


\section{REFERENCES}

[1] Lohmann, A. W., "Improvements relating to lenses and to variable optical lens systems formed by such lenses," British patent 998,191 (May 29, 1964).

[2] Lohmann, A. W., "Lentille de distance focale variable," French patent 1,398,351 (June 10, 1964).

[3] Lohmann, A. W., "Lente focale variabile," Italian patent 727,848 (June 19, 1964).

[4] Alvarez, L. W., "Two-element variable-power spherical lens," U.S. patent 3,305,294 (Dec. 3, 1964).

[5] Ledesma-Carrillo, L., Guzmán-Cabrera, R., Gómez-Sarabia, C. M., Torres-Cisneros, M., and Ojeda-Castañeda, J., "Tunable field depth: hyperbolic optical masks," Appl. Opt. 56 (1), A104-A114 (2017).

[6] Lohmann, A. W., “A new class of varifocal lenses,” Appl. Opt. 9 (7), 1669-1671 (1970).

[7] Dyson, J., "Circular and spiral diffraction gratings," Proc. R. Soc. Lond. A 248, 93-106 (1958).

[8] Bryngdahl, O., "Radial- and circular-fringe interferograms," J. Opt. Soc. Am. 63 (9), 1098-1104 (1973).

[9] Ojeda-Castaneda, J., Gómez-Sarabia, C. M., and Ledesma, S., "Tunable focalizers: axicons, lenses and axilenses," Proc. of SPIE 8833, 8833061-8833066 (2014).

[10] Ojeda-Castaneda, J., Ledesma, S. and Gómez-Sarabia, C. M., "Tunable Apodizers and Tunable Focalizers using helical pairs", Photonics Letters of Poland, Vol. 5 (1), 20-22 (2013).

[11] Ojeda-Castañeda, J., C. M. Gómez-Sarabia, C. M., and Ledesma, S., "Novel free-form optical pairs for tunable focalizers," J. Opt. 43 (2), 85-91 (2014).

[12] Bernet, S., M. Ritsch-Marte, M., "Optical device with a pair of diffractive optical elements," US Patent 0134869 A1 (2010).

[13] Grewe, A. and Stefan Sinzinger, S., "Efficient quantization of tunable helix phase plates," Opt. Lett. 41 (20) 4755-47 (2016).

[14] Grewe, A., Fesser, P. and Sinzinger, S., "Diffractive array optics tuned by rotation," Appl. Opt. 56 (1), A89A96 (2017).

[15] McLeod, J. H., “The Axicon: A New Type of Optical Element,” J. Opt. Soc. Am. 44 (8), 592-597 (1954).

[16] Davidson, N., Friesem, A. A. and Hasman, E., "Holographic axilens: high resolution and long focal depth," Opt. Lett. 16 (7), 523-525 (1991).

[17] Sochacki J., Bara, S., Jaroszewicz, Z., and Kolodziejczyk, A., "Phase retardation of the uniform-intensity axilens," Opt. Lett. 17 (1), 7-9 (1992).

[18] Jaroszewicz, Z., Kolodziejczyk, A., Mouriz, D., Gomez-Reino, C., "Spiral zone plates with arbitrary diameter of the dark spot in the centre of their focal point;" Opt. Commun. 114 (2), 1-8 (1995).

[19] Steel, W. H., "Axicons with spherical surfaces," in Optics in Metrology, P. Mollet, ed. (Pergamon, 1960), pp.181-193.

[20] McCutchen, C. W., "Convolution relation within the three-dimensional diffraction image," J. Opt. Soc. Am. A 8 (6), 868-870 (1991).

[21] Ojeda-Castañeda, J. and Gómez-Sarabia, C. M., "Tuning field depth at high resolution by pupil engineering," Advances in Optics and Photonics 7 (4), 814- 878 (2015).

[22] Ojeda-Castañeda, J., Landgrave, J. E. A., and Gómez-Sarabia, C. M., "Conjugate phase plate use in analysis of the frequency response of optical systems designed for extended depth of field ," Appl. Opt. 47 (22), E1-E7 (2008).

[23] Ojeda-Castaneda, J., Yépez-Vidal, E., García-Almanza, E., and Gómez-Sarabia, C. M., "Tunable Gaussian mask for extending the depth of field," Photon. Lett. Poland 4 (3), 115-117 (2012).

[24] Ojeda-Castaneda, J., Ledesma, L. and Valencia, R., "Tunable hyperbolic apodizer," Photon. Lett. of Poland, 7 (1), 11-13 (2015).

[25] Ojeda-Castañeda, J., Ledesma, S. and Gómez-Sarabia, C. M., "Helical apodizers for tunable hyper Gaussian masks," Proc. of SPIE 8842, 88420N1- 88420N6 (2014).

[26] Ojeda-Castañeda, J., Barragán-Chávez, A. L., and Cristina M. Gómez-Sarabia, C. M., "Aberration generators in tandem," Photon. Lett. of Poland, 7 (1), 8-10 (2015). 